\title{
The surprising external upturn of the Blue Straggler radial distribution in M55
}

\author{
B. Lanzoni ${ }^{2}$, E. Dalessandro ${ }^{2,3}$, S. Perina ${ }^{2,4}$, F.R. Ferraro ${ }^{2}$, R.T. Rood ${ }^{5}$, A. Sollima ${ }^{2}$ \\ ApJ accepted, 8 August, 07
}

\begin{abstract}
By combining high-resolution HST and wide-field ground based observations, in ultraviolet and optical bands, we study the Blue Straggler Star (BSS) population of the low density galactic globular cluster M55 (NGC 6809) over its entire radial extent. The BSS projected radial distribution is found to be bimodal, with a central peak, a broad minimum at intermediate radii, and an upturn at large radii. Similar bimodal distributions have been found in other globular clusters (M3, 47 Tucanae, NGC 6752, M5), but the external upturn in M55 is the largest found to date. This might indicate a large fraction of primordial binaries in the outer regions of M55, which seems somehow in contrast with the relatively low $(\sim 10 \%)$ binary fraction recently measured in the core of this cluster.
\end{abstract}

Subject headings: Globular clusters: individual (M55); stars: evolution - binaries: general - blue stragglers

\section{INTRODUCTION}

Blue straggler stars (BSS) are objects that the single mass stellar evolution theory is unable to explain. In the color-magnitude diagrams (CMDs) of evolved stellar populations, like globular clusters (GCs), they lie along an extension of the Main Sequence (MS), in a

\footnotetext{
${ }^{1}$ Based on observations with the NASA/ESA HST, obtained at the Space Telescope Science Institute, which is operated by AURA, Inc., under NASA contract NAS5-26555. Also based on WFI observations collected at the European Southern Observatory, La Silla, Chile.

${ }^{2}$ Dipartimento di Astronomia, Università degli Studi di Bologna, I-40127 Bologna, Italy

${ }^{3}$ Agenzia Spaziale Italiana, Centro di Geodesia Spaziale, I-75100 Matera, Italy

${ }^{4}$ INAF-Osservatorio Astronomico di Bologna, I-40127 Bologna, Italy

${ }^{5}$ Astronomy Department, University of Virginia, P.O. Box 400325, Charlottesville, VA, 22904
} 
region which is brighter and bluer than the Turn-Off (TO), where no stars are expected to be found. Their position in the CMD indicates that BSS are rejuvenated stars, with masses larger than the normal cluster stars (this is also confirmed by direct mass measurements; e.g. Shara et al. 1997). Thus, they are thought to have increased their initial mass during their evolution, and two main scenarios have been proposed for their formation: the collisional scenario (Hills \& Day 1976) suggests that BSS are the end-products of stellar mergers induced by collisions (COL-BSS), while in the mass-transfer (McCrea 1964; Zinn \& Searle 1976) scenario BSS form by the mass-transfer activity between two companions in a binary system (MT-BSS), possibly up to the complete coalescence of the two stars. Hence, understanding the origin of BSS in stellar clusters provides valuable insight both on the binary evolution processes, and on the effects of dynamical interactions on the (otherwise normal) stellar evolution.

The two formation mechanisms are likely to be at work simultaneously in every GC (see the case of M3 as an example; Ferraro et al. 1993, 1997), with a relative efficiency that probably depends on the local density (Fusi Pecci et al. 1992; Ferraro et al. 1999a; Bellazzini et al. 2002; Ferraro et al. 2003). In fact, since stellar collisions are most probable in high-density environments, COL-BSS are expected to be formed preferentially in the cluster cores, while MT-BSS can populate both the centre and the peripheries. Primordial binaries can in fact sink to the core due to dynamical friction and mass segregation processes, and "new" binaries can be formed in the cluster centers by gravitational encounters. To be completely clear with our terminology, a primordial binary which has sunk to the cluster center and is then driven to merge by stellar interactions (rather than by evolution of the more massive member of the binary or by magnetic braking as in the case of W Uma systems) is classified as a COL-BSS. Of course, in the case of a low-density cluster a fraction of primordial binaries evolving in isolation (hence classified as MT-BSS in our terminology) can well be present even in the cluster center.

One possibility for distinguishing between the two types of BSS is offered by highresolution spectroscopic studies. In fact, anomalous chemical abundances are expected at the surface of BSS resulting from MT activity (Sarna \& de Greve 1996), while they are not predicted in case of a collisional formation (Lombardi et al. 1995). Such studies have just become feasible, and the results found in the case of 47 Tucanae (47 Tuc; Ferraro et al.|2006a) are encouraging. The detection of unexpected properties of stars along standard evolutionary sequences (e.g., variability, anomalous population fractions, peculiar radial distributions, or a secondary MS) can help to estimate the fraction of binaries within a cluster (see, e.g., Bailyn 1994; Albrow et al. 2001; Bellazzini et al. 2002; Beccari et al. 2006b; Sollima et al. 2007), but such evidence does not directly allow the determination of the relative efficiency of the two BSS formation processes. Instead, the observational study of the BSS radial dis- 
tribution within the host clusters, complemented with suitable dynamical simulations, has proved to be a more widely applicable and powerful tool (see Ferraro 2006, for a review). Observations have shown that BSS are generally highly concentrated in the cluster cores, and in some cases, specifically in M3 (Ferraro et al. 1997), 47 Tuc (Ferraro et al. 2004), NGC 6752 (Sabbi et al. 2004), and M5 (Warren et al. 2006; Lanzoni et al. 2007a), their projected radial distribution is bimodal, i.e., their fraction with respect to the normal cluster populations (like horizontal branch or red giant branch stars) decreases to a minimum, and then rises again to larger values for increasing radii. Dynamical simulations suggest that the external rising branch cannot be due to COL-BSS generated in the core and kicked out by dynamical interactions (Mapelli et al. 2004). Instead, the bimodality of the radial distribution can be explained (Mapelli et al. 2004, 2006; Lanzoni et al. 2007a) by assuming that a non-negligible fraction ( $\gtrsim 20 \%-40 \%$ ) of the BSS population is made of MT-BSS (responsible for the external rising branch), with the balance being COL-BSS (mainly contributing to the central peak). The atypical case of $\omega$ Centauri (where the BSS radial distribution has been found to be flat; Ferraro et al. 2006b) can be explained if the mass segregation processes have not yet played a major role in this system, thus implying that it is populated by a vast majority of MT-BSS (Mapelli et al. 2006). These results demonstrate that detailed studies of the BSS radial distribution within GCs are powerful tools for better understanding the complex interplay between dynamics and stellar evolution in dense stellar systems. Extending this kind of investigation to a larger sample of GCs, with different structural and dynamical characteristics, is crucial for identifying the cluster properties that mainly affect the BSS formation mechanisms and their relative efficiency.

The present paper is devoted to the study of the BSS projected radial distribution in M55 (NGC 6809). Previous works have suggested that BSS in this cluster are more centrally concentrated than MS and sub-giant branch (SGB) stars (Mandushev et al. 1997; Zaggia et al. 1997, hereafter Z97), and that the BSS radial distribution is bimodal (Z97). These studies, however, were based only on partial coverage of the cluster area, while the wide-field observations used in this paper cover almost all of the entire cluster extension. In addition, we have sampled the core region with HST high-resolution observations both in the ultraviolet and in the optical bands, thus getting a more reliable and efficient detection of the BSS and of the normal cluster populations. In the following we present the observational data sets and the results obtained. We postpone to a forthcoming paper the theoretical interpretation of the BSS radial distribution by means of dynamical simulations, and a detailed comparison with all the other GCs studied with the same technique. 


\section{OBSERVATIONS AND DATA ANALYSIS}

\subsection{The Data Sets}

The present study is based on a combination of two different photometric data sets:

1. The high-resolution set - It consists of a series of HST-WFPC2 images of the cluster center (Prop. 10524, P.I. Ferraro), obtained through filter F255W (medium UV, for a total exposure time $t_{e x p}=2000 \mathrm{~s}$ ) and F336W (approximately corresponding to an $U$ filter, with $t_{\text {exp }}=1600 \mathrm{~s}$ ). To efficiently resolve the stars in the highly crowded central regions, the Planetary Camera has been pointed approximately on the cluster center, while the three Wide Field Cameras (WFC) have been used to sample the surrounding regions. The photometric reduction of the images was carried out using ROMAFOT (Buonanno et al. 1983), a package developed to perform accurate photometry in crowded fields and specifically optimized to handle under-sampled point spread functions (PSFs; Buonanno \& Iannicola 1989), as in the case of the WFC chips. Additional HST images of the cluster center, obtained with the ACS-Wide Field Channel (Prop. 10775, P.I. Sarajedini) have been retrieved from the ESO-STECF Science Archive. Only the short exposures (10 sec each) in filters F606W $(V)$ and $\mathrm{F} 814 \mathrm{~W}(I)$ have been used in the present work. The adopted data reduction procedure is described in detail in Sollima et al. (2007). The map of the ACS data set is shown in Figure 1, together with the WFPC2 and ACS fields of view (FoVs).

2. The wide-field set - A complementary set of public wide-field $B$ and $V$ images obtained with the Wide Field Imager (WFI) at the 2.2m ESO-MPI telescope was retrieved from the ESO Science Archive. Thanks to the wide $\left(34^{\prime} \times 34^{\prime}\right)$ FoV of WFI, these data almost cover the entire cluster extension (see Fig. 2, where the cluster is roughly centered on CCD \#7). The raw WFI images were corrected for bias and flat field, and the overscan regions were trimmed using IRAF1 tools. The PSF fitting procedure was then performed independently on each image using DoPhot (Schechter et al. 1993).

\subsection{Astrometry and Photometric Calibration}

The HST and WFI catalogs have been placed on the absolute astrometric system by adopting the procedure described in Ferraro et al. (2001, 2003). The new astrometric Guide

\footnotetext{
${ }^{1}$ IRAF is distributed by the National Optical Astronomy Observatory, which is operated by the Association of Universities for Research in Astronomy, Inc., under cooperative agreement with the National Science Foundation.
} 
Star Catalog (GSC-II2) was used to search for astrometric standard stars in the WFI FoV, and a specific cross-correlation tool has been employed to obtain an astrometric solution for each of the 8 CCDs. Several hundred GSC-II reference stars were found in each chip, thus allowing an accurate absolute positioning. Then, a few hundred stars in common between the WFI and the HST FoVs have been used as secondary standards to place the HST catalogs on the same absolute astrometric system. At the end of the procedure the global uncertainties in the astrometric solution are of the order of $\sim 0^{\prime \prime} .2$, both in right ascension $(\alpha)$ and declination $(\delta)$.

The photometric calibration of the optical $(B$ and $V$ ) magnitudes has been performed by using the Stetson Photometric Standard catalog 3 . After cross correlating the WFI and Stetson catalogs, we have used the stars in common for the calibration of the WFI $B$ and $V$ magnitudes. Then, the ACS $V$ magnitudes have been converted to the WFI system by using the stars in common. Since the Stetson standard field does not overlap with the ACS FoV, the calibration of the ACS I magnitudes has been performed by using the stars in common with the catalog of Desidera et al. (1998), after converting the latter to the Stetson photometric system. Finally, the WFPC2 $m_{255}$ and $U$ magnitudes have been calibrated to the Holtzman et al. (1995) zero-points. The resulting CMDs, both in the UV and optical bands, are shown in Figures 3 and 4 , respectively.

Unless otherwise specified, in the following analysis we adopt the combined HST catalog (ACS and WFPC2 data) for the cluster central regions (see Fig. 1), and the complementary WFI sample for the external parts (see Fig. 2).

\subsection{Center of Gravity, and Density Profile}

Given the absolute positions and the magnitudes of individual stars, the center of gravity $C_{\text {grav }}$ has been determined by averaging the coordinates $\alpha$ and $\delta$ of all stars brighter than $V=19$ lying in the FoV of WFI CCD \#7. We have chosen to use the WFI (instead of the $H S T$ ) data, because in such a loose cluster the FoV of the WFPC2 planetary camera is too small to provide an adequately large sample for the accurate determination of the center of gravity, while the ACS FoV is crossed by the gap between the two chips. Following the iterative procedure described in Montegriffo et al. (1995), we have determined $C_{\text {grav }}$ to be $\alpha(\mathrm{J} 2000)=19^{\mathrm{h}} 39^{\mathrm{m}} 59.54, \delta(\mathrm{J} 2000)=-30^{\circ} 57^{\prime} 45^{\prime \prime} .14$, with a $1 \sigma$ uncertainty of $0^{\prime \prime} .5$ in both

\footnotetext{
${ }^{2}$ Available at http://www-gsss.stsci.edu/Catalogs/GSC/GSC2/GSC2.htm.

${ }^{3}$ Available at http://www1.cadc-ccda.hia-iha.nrc-cnrc.gc.ca/community/STETSON/standards/.
} 
$\alpha$ and $\delta$. This value of $C_{\text {grav }}$ is located $\sim 2^{\prime \prime}$ south-east $\left(\Delta \alpha=2^{\prime \prime} .1, \Delta \delta=-1^{\prime \prime}\right.$. 1$)$ from that previously derived by Harris (1996) on the basis of the surface brightness distribution.

By exploiting the optimal combination of high-resolution and wide-field sampling provided by our observations, we have determined the projected density profile by direct star counts over the entire cluster radial extent, from $C_{\text {grav }}$ out to $\sim 1400^{\prime \prime} \sim 23^{\prime}$. To avoid biases due to incompleteness, we have considered only stars brighter than $V=19$ from the ACS and the complementary WFI catalogs (see Fig. 4). The brightest red giant branch (RGB) stars that are strongly saturated in the ACS data set have been excluded from the analysis, but since they are few in number and the ACS pixel scale is only of $0.05^{\prime \prime} /$ pixel, the effect on the resulting density profile is negligible. Following the procedure described in Ferraro et al. (1999a, 2004), we have divided the entire HST+WFI sample in 26 concentric annuli, each centered on $C_{\text {grav }}$ and split in an adequate number of sub-sectors. The number of stars lying within each sub-sector was counted, and the star density was obtained by dividing these values by the corresponding sub-sector areas. The stellar density in each annulus was then obtained as the average of the sub-sector densities, and its standard deviation was estimated from the variance among the sub-sectors. The radial density profile thus derived is shown in Figure 5, and the average of the three outermost $\left(r \gtrsim 17^{\prime}\right)$ measures has been used to

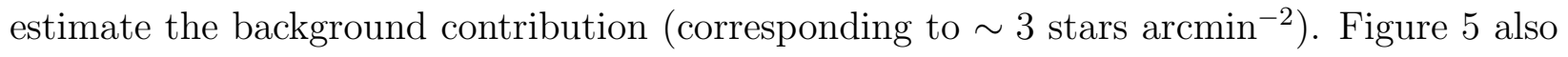
shows the best-fit mono-mass King model and the corresponding values of the core radius and concentration: $r_{c}=114^{\prime \prime}$ (with a typical error of $\sim \pm 2^{\prime \prime}$ ) and $c=1$, respectively. These values are in agreement with those quoted by McLaughlin \& van der Marel (2005, $r_{c}=126^{\prime \prime} 4$ and $c=0.93)$, and by Irwin \& Trimble $\left(1984, r_{c} \sim 120^{\prime \prime}\right.$ and $\left.c \sim 1\right)$. Concentration parameters as low as $\sim 0.8$ (as quoted, e.g., by Harris 1996, Z97) provide significantly worse fits to the observed profile. The difference with respect to (Z97, who also computed the surface density profile by direct star counts) is probably due to the fact that their ground-based observations are saturated at $V \lesssim 14$, and have a pixel scale much larger than that of ACS, so they have lost a number of faint stars in the central regions of the cluster. Assuming the distance modulus $(m-M)_{0}=13.82(d \sim 5.8 \mathrm{Kpc}$, Ferraro et al. 1999b), our value of $r_{c}$ corresponds to $\sim 3.2 \mathrm{pc}$. These values can then be used to redetermine the other structure parameters of the cluster. By assuming $\mu_{0}=19.13 \mathrm{mag} / \mathrm{arcsec}^{2}$ as the central surface brightness (Harris 1996), and $E(B-V)=0.07$ as reddening (Ferraro et al. 1999b), we estimate that the extinction-corrected central surface brightness of the cluster is $\mu_{V, 0}(0) \simeq 18.91$ mag/arcsec ${ }^{2}$. Following the procedure described in Djorgovski (1993, see also Beccari et al. 2006a), we derive $\log \nu_{0} \simeq 2.23$, where $\nu_{0}$ is the central luminosity density in units of $L_{\odot} \mathrm{pc}^{-3}$. By assuming a mass-to-light ratio $M / L_{V}=3$, the derived central mass density measured in $M_{\odot} / \mathrm{pc}^{3}$ is $\log \rho_{0}=2.7$, which is a factor $\sim 1.6$ higher than that quoted by Pryor \& Meylan (1993). This value corresponds to $n_{0} \simeq 1000 \mathrm{stars}^{\mathrm{pc}} \mathrm{c}^{-3}$ if a mean stellar 
mass of $0.5 M_{\odot}$ is assumed.

\section{CLUSTER POPULATION SELECTION}

\subsection{The BSS Population}

At UV wavelengths BSS are among the brightest objects in a GC, and RGB stars are particularly faint. By combining these advantages with the high-resolution capability of HST, the usual problems associated with photometric blends and stellar crowding are minimized, and BSS can be most reliably recognized and separated from the other populations in the UV CMDs (see also Ferraro et al. 2004). For these reasons our primary criterion for the definition of the BSS sample is based on the position of stars in the $\left(m_{255}, m_{255}-U\right)$ plane. In order to avoid incompleteness bias and to limit the possible contamination from TO and SGB stars, we have chosen a limiting magnitude of $m_{255}=18.5$ (roughly 1 magnitude brighter than the cluster TO). The adopted selection box and the resulting 12 BSS identified in the UV plane are shown in Figure 3. Once selected in the UV CMD, the BSS lying in the field in common with the ACS sample have been used to define the selection box and the limiting magnitude in the $(V, V-I)$ plane. The latter is $V \simeq 17.5$, and the adopted selection box is shown in the left-hand panel of Figure 4. One of the BSS candidates (that lies close to the reddest edge of the box) has been rejected from the sample on the basis of its position in the UV plane, where it is $\sim 0.2$ magnitudes fainter than the adopted $m_{255}$ limit and has a color of $m_{255}-U=1$, thus clearly belonging to the SGB star population. A total of 24 BSS have been identified within the ACS selection box, of which 11 are in common with the WFPC2 sample. Finally, in order to select the BSS population in the complementary WFI data set, we have adopted the same $V$ magnitude limits as for the ACS sample. Since field star contamination is critical in M55, particularly in the external regions of the cluster, the definition of the $B-V$ color edges of the selection box has required a detailed study of the color-magnitude distribution of field stars. To do this, we have exploited both the outermost portion of the WFI observations (beyond the tidal radius), and the Galaxy model of Robin et al. (2003) in the direction of the cluster. In order to limit both the risk of field star and SGB blend contamination, we pick $B-V \simeq 0.41$ as a conservative value for the red-edge of the BSS selection box. As blue limit, we have chosen $B-V \simeq 0.08$. The adopted selection box in the $(V, B-V)$ plane is shown in the right-hand panel of Figure 4, and the number of enclosed BSS is 38 .

Since M55 is known to harbor a large population of SX Phoenicis (SX Phe) variables in the BSS region (Pych et al. 2001), we have cross-correlated the SX Phe catalog with our data set. All of the 24 SX Phe identified by Pych et al. (2001) are contained in our sample 
(see triangles in Figs. 3 and 4), and all but two lie within our BSS selection boxes. The two outliers (V21 and V26 in their catalog) are indeed only slightly redder than the adopted limits, and most likely are genuine BSS (in fact, BSS frequently show the pulsating properties of SX Phe stars; see, e.g., Mateo 1996). Thus, they have been also included in our BSS sample. The SX Phe population of M55 is truly remarkable, second only to the always weird

$\omega$ Centauri (Kaluzny et al. 2004). Considering that we have identified 56 BSS within the FoV in common with Pych et al. (2001) and that 24 of them are SX Phe variables, we see that almost half $(43 \%)$ of the BSS in M55 are pulsating.

The coordinates and magnitudes of all the selected BSS (65) are listed in Table 1, and their projected spatial distribution is shown in Figures 1 and 2, Two candidate BSS (namely, BSS 64 and 65 in Table 1) lie at $r>r_{t}$. Since Z97 suggest that there is tidal distortion in the north-east direction, these BSS could be part of a cluster tidal tail. However, our observations do not indicate any significant distortion in the cluster stellar distribution (although a more extended mapping of the surrounding regions might be needed), and we therefore conclude that they probably are field stars. Thus, they are not encircled in the right-hand panel of Figure 4, and have not been considered in the following analysis.

No quantitative comparison between our selected BSS population and that presented in Z97 is possible, since they provide neither selection criteria nor the coordinates of the identified BSS. Within the FoV (the inner $4^{\prime} \times 4^{\prime}$ ) in common with Mandushev et al. (1997), we find 33 BSS; for comparison, by using the published BSS magnitudes, we have verified that 30 of their stars are included in our BSS selection boxes, thus showing a very good agreement between the two studies. The remaining 44 BSS identified by these authors are fainter and/or redder than the limits adopted in the present work.

\section{The Reference Populations}

To study the BSS projected radial distribution and detect possible peculiarities, a reference population which is representative of the normal cluster stars must be properly defined. For this purpose we have chosen the horizontal branch (HB) and the RGB populations, both of which are expected to have a non-peculiar radial distribution within the cluster.

The adopted HB selection boxes in the optical CMDs are shown in Figure 4, and are designed to include the bulk of this population. The box in the UV plane defined by the stars in common between the ACS and the WFPC2 samples is shown in Figure 3, and confirms the suitability of the adopted selection. By cross-correlating the coordinates of our catalog with the catalog of RR Lyrae variables detected by Olech et al. (1999) we have identified 10 
stars (filled squares in Fig. 4) out of a total of 13, the remaining three falling in the gaps of the WFI chips. The few RR Lyrae that lie outside the selection boxes have been also included in our HB sample. Thus, the total number of selected HB stars is 237 (78 in the ACS data set, and 159 in the WFI sample) 4

In selecting the RGB sample, we have considered only the magnitude range $15.8 \leq V \leq$ 17.5 (the same adopted for the BSS), since the brightest RGB stars are saturated in the ACS observations. We have drawn narrow selection boxes around the RGB mean ridge line in the CMDs, in order to limit the contamination by field stars. The adopted boxes are shown in Figure 4, and the resulting number of RGB stars found at $r \leq r_{t}$ is 1504 .

\subsection{Field Contamination}

As apparent from the right-hand panel of Figure 4, field star contamination is a critical issue in the study of M55, particularly for the cluster outer regions. In order to estimate the impact of the field contamination on the cluster population selections, we have considered the CMD in the outermost $\left(r>r_{t}\right)$ portion of the WFI data set. By considering that the sampled area is of $\sim 252 \operatorname{arcmin}^{2}$, counts of stars within the adopted BSS, HB, and RGB selection boxes yield the number densities of field stars contaminating the selected cluster populations. As a further check, we have performed the same analysis on the synthetic data Galaxy model of Robin et al. (2003) in the $B$ and $V$ bands, considering a much larger area (1 square degree) in the direction of M55. The number densities derived from the two methods agree within a factor of $\sim 2-3$, and we have finally adopted densities obtained from the Galaxy model, because of the much larger sampled area. The estimated contamination is roughly 8, 4, and 550 field stars per square degree for the selected populations of BSS, HB, and RGB stars, respectively. By using the $V$ and $I$ data of the synthetic Galaxy model, we have verified that the same values are also appropriate for decontaminating the cluster populations in the inner $202^{\prime \prime} \times 202^{\prime \prime}$ (the ACS FoV) of our sample, where the selection has been performed in these photometric bands. The quoted values have been adopted in the following analysis to statistically decontaminate the star counts.

\footnotetext{
${ }^{4}$ Only one object lying in the HB box has been excluded because it is located at $r>r_{t}$
} 


\section{THE BSS PROJECTED RADIAL DISTRIBUTION}

As for other clusters studied in a similar way (see references in Lanzoni et al. 2007b), we have searched for possible peculiarities in the BSS radial distribution by comparing it with that of $\mathrm{HB}$ and RGB stars, that are expected to be distributed as "normal" cluster stars.

We have used the Kolmogorov-Smirnov (KS) test to search for statistical differences between the cumulative projected radial distributions of BSS and HB stars (the comparison with the RGB population has not been performed because of the non-negligible degree of field star contamination). As shown in Figure 6, BSS appear to be more concentrated than normal cluster stars within $\sim 300^{\prime \prime}$ from the center, and less concentrated outward. The statistical significance of this result, however, is rather poor: the overall KS probability that BSS and HB stars are not extracted from the same parent population is $\sim 0.90$ (corresponding to $\sim 1.6 \sigma$ significance level). If the analysis is restricted to the inner $300^{\prime \prime}$, BSS are more concentrated than HB stars at $\sim 1.9 \sigma$ level. For $r>300^{\prime \prime}$, where less than $20 \%$ of the total BSS and HB populations are located, the BSS are less concentrated at the $3 \sigma$. A similar trend, with a similar statistical significance, was also found by Z97, who, however, performed the comparison with the RGB population.

For a more quantitative analysis, the surveyed area has been divided into 5 concentric annuli (see Figs. 1 and 2), and the number of BSS, HB, and RGB stars $\left(N_{\mathrm{BSS}}, N_{\mathrm{HB}}\right.$, and $N_{\mathrm{RGB}}$, respectively) within each annulus has been counted. The resulting number counts have then been corrected for field contamination by taking into account the fraction of annulus area effectively sampled by our observations, and the estimated density of contaminating field stars for each population (see previous section). The values thus obtained are listed in Table 2 and have been used to compute the specific frequencies $N_{\mathrm{BSS}} / N_{\mathrm{HB}}, N_{\mathrm{BSS}} / N_{\mathrm{RGB}}$, and $N_{\mathrm{HB}} / N_{\mathrm{RGB}}$. Since the number of stars in any post-MS stage is proportional to the duration of the evolutionary phase itself (Renzini \& Buzzoni 1986), the specific frequencies $N_{\mathrm{HB}} / N_{\mathrm{RGB}}$ is expected to be constant and equal to the ratio between the evolutionary time scales of the HB phase and of the RGB portion in the magnitude range $15.8 \leq V \leq 17.5$, where the stars have been counted. In order to verify this, we have used the BAST:5 evolutionary model library (Pietrinferni et al. 2006, and reference therein), selecting the $\alpha$-enhanced low-temperature opacities tracks computed for metallicities $[\mathrm{Fe} / \mathrm{H}]=-1.84$ and $[\mathrm{M} / \mathrm{H}]=-1.49$ (the closest to the observed values $[\mathrm{Fe} / \mathrm{H}]=-1.61$ and $[\mathrm{M} / \mathrm{H}]=-1.41$; Ferraro et al. 1999b). From these models we have estimated that the time spent by a $0.8 M_{\odot}$ star along the RGB sequence in the range $15.8 \leq V \leq 17.5$ is $t_{\mathrm{RGB}} \sim 0.6 \mathrm{Gyr}$, while the duration of the HB phase for a 0.63

\footnotetext{
${ }^{5}$ Available at http://www.te.astro.it/BASTI/index.php
} 
$M_{\odot}$ is $t_{\mathrm{HB}} \sim 0.09 \mathrm{Gyr}$; thus, $t_{\mathrm{HB}} / t_{\mathrm{RGB}} \simeq 0.15$, in good agreement with the observed value of the $N_{\mathrm{HB}} / N_{\mathrm{RGB}}$ ratio (see the dotted line in the lower panel of Figure 7). A very similar result is also found by using the theoretical stellar tracks of the Pisa Evolutionary Library 6 (see references in Cariulo et al. 2004), and it ensures that the selected (and decontaminated) HB and RGB populations are indeed representative of the normal cluster stars. As for the BSS, the specific frequency $N_{\mathrm{BSS}} / N_{\mathrm{HB}}$ shows a completely different projected radial distribution, with a clearly bimodal behavior: from a central value of $\sim 0.4$, the BSS specific frequency decreases to a minimum at about $4 r_{c}$, and rises again at larger radii. A very similar trend (with the central peak at $\sim 0.07$ ) is also found for $N_{\mathrm{BSS}} / N_{\mathrm{RGB}}$, in agreement with Z97.

By integrating the density profile from the best-fit King model (see Sect 2.3), and assuming the values of central surface brightness, reddening and distance modulus quoted in Sect. 2.3, we have also computed the luminosity sampled in each annulus $\left(L^{\text {samp }}\right)$, and the total sampled luminosity $\left(L_{\mathrm{tot}}^{\mathrm{samp}}\right)$ taking into account the incomplete spatial coverage of the most external annulus (see Fig. 2). The resulting ratios between these two quantities in each annulus are listed in Table 2, and have been used to compute the double normalized ratio (see Ferraro et al. 1993):

$$
R_{\mathrm{pop}}=\frac{\left(N_{\mathrm{pop}} / N_{\mathrm{pop}}^{\mathrm{tot}}\right)}{\left(L^{\mathrm{samp}} / L_{\mathrm{tot}}^{\mathrm{samp}}\right)},
$$

where pop = BSS, HB, RGB.

The radial trend of $R_{\mathrm{HB}}$ (as well as that of $R_{\mathrm{RGB}}$ ) is essentially constant, with a value close to unity (see Fig. 8). This is just what expected on the basis of the stellar evolution theory, which predicts that the fraction of stars in any post-MS evolutionary stage is strictly proportional to the fraction of the sampled luminosity (Renzini \& Fusi Pecci 1988). Conversely, the trend of $R_{\mathrm{BSS}}$ is bimodal and indicates that, with respect to the sampled luminosity, the fraction of BSS is higher in the central regions and (particularly) in the cluster outskirts, and smaller at intermediate radii, with respect to the fraction of normal cluster stars

\section{DISCUSSION}

We have found that the BSS projected radial distribution in M55 is bimodal, i.e., peaked in the center, decreasing at intermediate radii, and rising again in the exterior. This is in

\footnotetext{
${ }^{6}$ Available at http://astro.df .unipi.it/SAA/PEL/ZO.html
} 
agreement with the findings of Z97 from the analysis of a much smaller fraction of the cluster, and puts their result on much more solid statistical basis.

Such a bimodality is similar to that found in M3 (Ferraro et al. 1997), 47 Tuc (Ferraro et al. 2004), NGC 6752 (Sabbi et al. 2004), and M5 (Warren et al. 2006; Lanzoni et al. 2007a). As in those GCs, also in M55 the position of the observed minimum approximately corresponds to the radius of avoidance $r_{\text {avoid }}$ of the system, i.e., the radius within which all the stars as massive as $1.2 M_{\odot}$ (which is assumed to be the typical BSS mass) are expected to have already sunk to the core due to dynamical friction and mass segregation processes. In fact, by using the dynamical friction timescale formula (from, e.g., Mapelli et al. 2006) with the best-fit King model and the central stellar density presented in Sect. 2.3, and assuming $\sigma \simeq 4.9 \mathrm{~km} \mathrm{~s}^{-1}$ as the central velocity dispersion (Pryor \& Meylan 1993), and $12 \mathrm{Gyr}$ as the cluster age, we estimate that $r_{\text {avoid }} \simeq 4.5 r_{c}$, in reasonable agreement with the position of the observed minimum.

The BSS specific frequency in the center of M55 $\left(N_{\mathrm{BSS}} / N_{\mathrm{HB}} \simeq 0.4\right)$ is also similar to that measured in the other bimodal GCs (cfr. Fig.7, with Fig. 12 of Lanzoni et al. 2007a, and see also Lanzoni et al. 2007b), where the central peak of the distribution is found to be mainly generated by COL-BSS (see also Mapelli et al. 2006). However, the central density in M55 is much lower (by a factor of 100 or more), and stellar collisions are expected to be less important in this system. Indeed, the cluster central density is quite similar to that of NGC 288 (only a factor of two higher), where most of the central BSS are thought to be MT-BSS (Bellazzini et al. 2002). A remarkable difference in the central value of $N_{\mathrm{BSS}} / N_{\mathrm{HB}}$ in these two low density clusters is however apparent. In fact, by considering only the brightest portion of the BSS population in NGC 288, Ferraro et al. (2003) measured $N_{\mathrm{BSS}} / N_{\mathrm{HB}} \simeq 1$, which is the highest BSS frequency ever found in a GC, together with that of M80 (Ferraro et al. 1999a), and is more than twice that of M55. What is the origin of this difference? One possibility is a different primordial binary fraction. However, Sollima et al. (2007) have recently estimated that the binary fractions in the core of the two clusters are the same $(\sim 10 \%)$. Another possibility is a substantial difference in the collision rate. By using equation (14) from Leonard (1989), we estimate that the central binary-binary collision rate in M55 is only a factor of $\sim 2$ higher than that in NGC 288. Moreover, the binary survival rate (defined as the ratio between the formation and destruction rates; see Verbunt 2003) is about twice as high in M55 than in NGC 288. Thus, our results indicate that two clusters with similar environments (and collision rates) and similar primordial binary content can produce quite different central BSS populations. Unfortunately, the BSS study in NGC 288 was restricted to two WFPC2 frames, and an investigation covering the entire cluster extension is urged in order to compare the global BSS population and its radial distribution in the two systems. 
Compared to the other bimodal GCs, the external rising branch in M55 is much more prominent. It is the largest upturn found to date $\left(N_{\mathrm{BSS}} / N_{\mathrm{HB}} \simeq 0.8 \pm 0.4\right.$ compared to the previous maximum value of $\simeq 0.25 \pm 0.11$, found in 47 Tuc). This is even more surprising if we consider that only $10 \%$ of the total cluster light is contained between $r_{\text {avoid }}$ and $r_{t}$ in M55, while it amounts to 32\% in the case of 47 Tuc. As discussed in Mapelli et al. (2006, see also Lanzoni et al. 2007a), the external rising branch is thought to be made of MT-BSS, generated in binary systems evolving in isolation in the cluster outskirts (this finding is also confirmed by the recent N-body simulations of Hurley et al. 2007). Thus, such a prominent upturn of the BSS distribution would imply a significantly higher primordial binary fraction in M55, compared to the other GCs. This seems in contrast with the results of Sollima et al. (2007), who measured the binary fractions in the core of 13 galactic GCs and found that M55 has one of the lowest fractions $(\sim 10 \%)$, with respect to the others, which range up to $\sim 50 \%$ (in Terzan 7 ). However a better understanding of the evolution of the binary fraction in the core, as a function of the cluster dynamical age, is needed to better address this point. In fact, the theoretical expectations for the time evolution of the core binary fraction are still controversial: while Ivanova et al. (2005) suggest that such a fraction significantly decreases in time, the opposite trend is found by Hurley et al. (2007). Moreover, since a careful investigation of the BSS radial distribution has not yet been performed in any of the other remaining clusters studied by Sollima et al. (2007), a comprehensive comparison of the BSS population properties in these systems is not yet possible.

The nature of the central BSS and of those producing the external rising branch in M55 is thus an open question. Appropriate dynamical simulations and detailed spectroscopic studies (see, e.g., Ferraro et al. 2006a) are therefore urged. We defer such studies to a forthcoming paper, where the results of our entire sample of clusters will be compared and discussed.

This research was supported by Agenzia Spaziale Italiana under contract ASI-INAF I/023/05/0, by the Istituto Nazionale di Astrofisica under contract PRIN/INAF 2006, and by the Ministero dell'Istruzione, dell'Università e della Ricerca. RTR is partially funded by NASA through grant number GO-10524 from the Space Telescope Science Institute. ED is supported by a grant financed by ASI. We also acknowledge J. Kaluzny for having provided us with the information about the FoV used in Pych et al. (2001) and Olech et al. (1999). This research used the facilities of the Canadian Astronomy Data Centre operated by the National Research Council of Canada with the support of the Canadian Space Agency. 


\section{REFERENCES}

Albrow, M. D., et al. 2001, ApJ, 559, 1060

Bailyn, C. D. 1994, AJ, 107, 1073

Beccari, G., Ferraro, F. R., Possenti, A., Valenti, E., Origlia, L., \& Rood, R. T. 2006a, AJ, 131,2551

Beccari, G., Ferraro, F. R., Lanzoni, B., \& Bellazzini, M., 2006b, ApJ, 652, L121

Bellazzini, M., Fusi Pecci, F., Messineo, M., Monaco, L., \& Rood, R. T. 2002, AJ, 123, 1509

Buonanno, R., Buscema, G., Corsi, C. E., Ferraro, I., \& Iannicola, G. 1983, A\&A, 126, 278

Buonanno, R., \& Iannicola, G. 1989, PASP, 101, 294

Cariulo, P., Degl'Innocenti, S., \& Castellani, V. 2004, A\&A, 421, 1121

Desidera, S. 1998, Master's thesis, Padua Univ.

Djorgovski, S. 1993, in ASPC Conf. Ser. 50, Structure and Dynamics of Globular Clusters, ed. S. G. Djorgovski \& G. Meylan (San Francisco: ASP), 373D

Ferraro, F. R., Fusi Pecci, F., Cacciari, C., Corsi, C., Buonanno, R., Fahlman, G. G., \& Richer, H. B. 1993, AJ, 106, 2324

Ferraro, F. R., Paltrinieri, B., Fusi Pecci, F., Cacciari, C., Dorman, B., Rood, R. T., Buonanno, R., Corsi, C. E., Burgarella, D., \& Laget, M., 1997, A\&A, 324, 915

Ferraro, F. R., Paltrinieri, B., Rood, R. T., \& Dorman, B. 1999a, ApJ 522, 983

Ferraro F. R., Messineo M., Fusi Pecci F., De Palo M. A., Straniero O., Chieffi A.,\& Limongi M. 1999b, AJ, 118, 1738

Ferraro, F. R., D’Amico, N., Possenti, A., Mignani, R. P., \& Paltrinieri, B. 2001, ApJ, 561, 337

Ferraro, F. R., Sills, A., Rood, R. T., Paltrinieri, B., \& Buonanno, R. 2003, ApJ, 588, 464

Ferraro, F. R., Beccari, G., Rood, R. T., Bellazzini, M., Sills, A., \& Sabbi, E. 2004, ApJ, 603,127

Ferraro, F. R., 2006, in Resolved Stellar Populations, ASP Conference Series, D. VallsGabaud \& M. Chaves Eds., astro-ph/0601217 
Ferraro, F. R., et al. 2006a, ApJ, 647, L53

Ferraro, F. R., Sollima, A., Rood, R. T., Origlia, L., Pancino, E., \& Bellazzini, M. 2006b, ApJ, 638, 433

Fusi Pecci, F., Ferraro, F. R., Corsi, C. E., Cacciari, C., \& Buonanno, R. 1992, AJ, 104, 1831

Harris, W.E. 1996, AJ, 112, 1487

Hills, J. G., \& Day, C. A. 1976, Astrophys. Lett., 17, 87

Holtzman, J. A., Burrows, C. J., Casertano, S., Hester, J. J., Trauger, J. T., Watson, A. M., \& Worthey, G. 1995, PASP, 107, 1065

Hurley, J. R., Aarseth, S. J., \& Shara, M. M. 2007, in press on ApJ(astro-ph/0704.0290)

Irwin, M. J., \& Trimble, V. 1984, AJ, 89, 83

Ivanova, N., Belczynski, K., Fregeau, J. M., \& Rasio, F. A. 2005, MNRAS, 358, 572

Kaluzny J., Olech A., Thompson I. B., Pych W., Krzemiński W., \& Schwarzenberg-Czerny A. 2004, A\&A, 424, 1101

Lanzoni, B., Dalessandro, E., Ferraro, F. R., Mancini, C., Beccari, G., Rood, R. T., Mapelli, M., \& Sigurdsson, S. 2007a, in press on ApJ(astro-ph/0704.0139)

Lanzoni, B., et al. 2007b, ApJ, 663, 1040

Leonard, P. J. T. 1989, AJ, 98, 217

Lombardi, J. C. Jr., Rasio, F. A., \& Shapiro, S. L. 1995, ApJ, 445, L117

Mandushev, G. I., Fahlman, G. G., Richer, H. B., \& Thompson, I. B. 1997, AJ, 114, 1060

Mapelli, M., Sigurdsson, S., Colpi, M., Ferraro, F. R., Possenti, A., Rood, R. T., Sills, A., \& Beccari, G. 2004, ApJ, 605, L29

Mapelli, M., Sigurdsson, S., Ferraro, F. R., Colpi, M., Possenti, A., \& Lanzoni, B. 2006, MNRAS, 373, 361

Mateo M., in "The origins, evolution, and destinies of binary stars in clusters", E. F. Milone \& J. C. Mermilliod Eds. 1996, ASPC, 90, 346

McLaughlin, D. E., \& van der Marel, R. P. 2005, ApJS, 161, 304 
McCrea, W. H. 1964, MNRAS, 128, 147

Montegriffo, P., Ferraro, F. R., Fusi Pecci, F., \& Origlia, L. 1995, MNRAS, 276, 739

Olech, A., Kaluzny, J., Thompson, I. B., Pych, W., Krzeminski, W., \& Shwarzenberg-Czerny, A. 1999, AJ, 118, 442

Pietrinferni, A., Cassisi, S., Salaris, M., \& Castelli, F. 2006, ApJ, 642, 797

Pryor C., \& Meylan G., 1993, Structure and Dynamics of Globular Clusters. Proceedings of a Workshop held in Berkeley, California, July 15-17, 1992, to Honor the 65th Birthday of Ivan King. Editors, S.G. Djorgovski and G. Meylan; Publisher, Astronomical Society of the Pacific, Vol. 50, 357

Pych, W., Kaluzny, J., Krzeminski, W., Schwarzenberg-Czerny, A., \& Thompson, I. B. 2001, A\&A, 367, 148

Renzini, A., \& Buzzoni, A. 1986, ASSL Vol. 122: Spectral Evolution of Galaxies, 195

Renzini, A., \& Fusi Pecci, F. 1988, ARA\&A, 26, 199

Robin, A. C., Reylé, C., Derrière, S., \& Picaud, S. 2003, A\&A, 409, 523

Sabbi, E., Ferraro, F. R., Sills, A., \& Rood, R. T., 2004, ApJ 617, 1296

Sarna, M. J., \& de Greve, J. P. 1996, QJRAS, 37, 11

Schechter, P. L., Mateo, M., \& Saha, A. 1993, PASP, 105, 1342

Shara, M. M., Saffer, R. A., \& Livio, M. 1997, ApJ, 489, L59

Sollima, A., Beccari., G., Ferraro, F. R., Fusi Pecci, F., \& Sarajedini, A., 2007, in press on MNRAS (astro-ph/0706.2288)

Verbunt, F. 2003, in ASP Conf. Ser. 296, New Horizons in Globular Cluster Astronomy, ed. G. Piotto et al. (San Francisco: ASP), 245

Warren, S. R., Sandquist, E. L., \& Bolte, M., 2006, ApJ 648, 1026 (W06)

Zaggia, S. R., Piotto, G., \& Capaccioli, M., 1997, A\&A, 327, 1004 (Z97)

Zinn, R., \& Searle, L. 1976, ApJ, 209, 734 
Table 1. The BSS Population of M55

\begin{tabular}{|c|c|c|c|c|c|c|c|c|}
\hline Name & $\begin{array}{c}\text { RA } \\
\text { [degree] }\end{array}$ & $\begin{array}{c}\text { DEC } \\
\text { [degree] }\end{array}$ & $m_{255}$ & $\mathrm{U}$ & B & V & I & SX Phe \\
\hline BSS 1 & 294.9998920 & -30.9667245 & 18.26 & 17.47 & - & - & - & - \\
\hline BSS 2 & 294.9954953 & -30.9396261 & 18.08 & 17.18 & - & 16.85 & 16.26 & - \\
\hline BSS 3 & 295.0121689 & -30.9581611 & 17.05 & 16.57 & - & 16.14 & 15.71 & - \\
\hline BSS 4 & 294.9982015 & -30.9483228 & 17.56 & 17.05 & - & 16.66 & 16.28 & - \\
\hline BSS 5 & 295.0166912 & -30.9704646 & 17.62 & 17.14 & - & 16.69 & 16.33 & - \\
\hline BSS 6 & 295.0193344 & -30.9660591 & 17.84 & 17.28 & - & 16.81 & 16.45 & - \\
\hline BSS 7 & 295.0045478 & -30.9669382 & 18.01 & 17.55 & - & 16.91 & 16.70 & - \\
\hline BSS 8 & 295.0033265 & -30.9834341 & 18.43 & 17.64 & - & 17.19 & 16.77 & - \\
\hline BSS 9 & 295.0104327 & -30.9803687 & 18.41 & 17.82 & - & 17.42 & 16.93 & - \\
\hline BSS 10 & 295.0122305 & -30.9747912 & 17.62 & 17.13 & - & 16.61 & 16.15 & V41 \\
\hline BSS 11 & 295.0040550 & -30.9659563 & 18.29 & 17.73 & - & 17.30 & 16.81 & V31 \\
\hline BSS 12 & 294.9902115 & -30.9506018 & 18.17 & 17.60 & - & 17.37 & 16.90 & V19 \\
\hline BSS 13 & 294.9849393 & -30.9719486 & - & - & - & 15.87 & 15.76 & - \\
\hline BSS 14 & 294.9748793 & -30.9741471 & - & - & - & 16.41 & 16.09 & - \\
\hline BSS 15 & 294.9858796 & -30.9600404 & - & - & - & 16.85 & 16.60 & - \\
\hline BSS 16 & 294.9702800 & -30.9607749 & - & - & - & 17.08 & 16.68 & - \\
\hline BSS 17 & 295.0254214 & -30.9727945 & - & - & - & 16.78 & 16.30 & - \\
\hline BSS 18 & 295.0100545 & -30.9422429 & - & - & - & 16.75 & 16.41 & - \\
\hline BSS 19 & 295.0214094 & -30.9804905 & - & - & - & 17.31 & 16.82 & - \\
\hline BSS 20 & 294.9951572 & -30.9710352 & - & - & - & 16.78 & 16.31 & V38 \\
\hline BSS 21 & 294.9921499 & -30.9759958 & - & - & - & 17.04 & 16.62 & V32 \\
\hline BSS 22 & 295.0285621 & -30.9424951 & - & - & - & 17.05 & 16.58 & V18 \\
\hline BSS 23 & 294.9788871 & -30.9728224 & - & - & - & 17.12 & 16.65 & V20 \\
\hline BSS 24 & 294.9751258 & -30.9689860 & - & - & - & 17.14 & 16.69 & $\mathrm{~V} 27$ \\
\hline BSS 25 & 294.9941390 & -30.9568945 & - & - & - & 17.20 & 16.78 & V42 \\
\hline BSS 26 & 294.9927055 & -30.9852788 & - & - & - & 15.84 & 15.20 & V21 \\
\hline BSS 27 & 294.9793701 & -31.0208092 & - & - & 16.17 & 15.92 & - & - \\
\hline BSS 28 & 294.7966919 & -31.0010357 & - & - & 16.21 & 16.11 & - & - \\
\hline BSS 29 & 295.0544434 & -30.8069954 & - & - & 16.23 & 16.10 & - & - \\
\hline BSS 30 & 295.0268860 & -30.9911098 & - & - & 16.30 & 16.01 & - & - \\
\hline BSS 31 & 295.0368652 & -30.9847641 & - & - & 16.60 & 16.47 & - & - \\
\hline BSS 32 & 295.0367126 & -30.9545650 & - & - & 16.60 & 16.35 & - & - \\
\hline BSS 33 & 295.0966492 & -30.9473000 & - & - & 16.61 & 16.21 & - & - \\
\hline BSS 34 & 294.9940796 & -30.9063625 & - & - & 16.63 & 16.36 & - & - \\
\hline BSS 35 & 294.9552917 & -30.9421539 & - & - & 16.77 & 16.43 & - & - \\
\hline BSS 36 & 295.0687561 & -30.9846306 & - & - & 16.81 & 16.52 & - & - \\
\hline
\end{tabular}


Table 1 - Continued

\begin{tabular}{lcccccccc}
\hline \hline Name & RA & $\begin{array}{c}\text { DEC } \\
\text { [degree] }\end{array}$ & $m_{255}$ & U & B & V & I & SX Phe \\
& Bdegree] & & & & & & \\
\hline BSS 38 & 295.0217285 & -30.9895248 & - & - & 16.87 & 16.58 & - & - \\
BSS 39 & 294.7169495 & -30.9712677 & - & - & 16.93 & 16.76 & - & - \\
BSS 40 & 294.9922485 & -30.6695671 & - & - & 17.07 & 16.78 & - & - \\
BSS 41 & 295.0174561 & -30.9149532 & - & - & 17.22 & 16.86 & - & - \\
BSS 42 & 294.7818298 & -31.0331841 & - & - & 17.32 & 16.97 & - & - \\
BSS 43 & 294.7232361 & -31.0292740 & - & - & 17.34 & 17.15 & - & - \\
BSS 44 & 294.9502563 & -30.7848854 & - & - & 17.39 & 16.98 & - & - \\
BSS 45 & 294.9739380 & -31.0131721 & - & - & 17.41 & 17.09 & - & - \\
BSS 46 & 295.0329895 & -30.9473553 & - & - & 17.62 & 17.30 & - & - \\
BSS 47 & 294.6565247 & -31.0778027 & - & - & 17.64 & 17.43 & - & - \\
BSS 48 & 294.9787292 & -30.9204979 & - & - & 17.72 & 17.38 & - & - \\
BSS 49 & 294.9926758 & -30.9187489 & - & - & 17.81 & 17.47 & - & - \\
BSS 50 & 294.9646912 & -30.9394836 & - & - & 16.42 & 16.13 & - & V25 \\
BSS 51 & 294.9772644 & -30.9996738 & - & - & 16.69 & 16.39 & - & V33 \\
BSS 52 & 294.9597473 & -30.9203262 & - & - & 16.97 & 16.64 & - & V35 \\
BSS 53 & 294.9522705 & -30.9460793 & - & - & 17.02 & 16.77 & - & V36 \\
BSS 54 & 295.0324402 & -31.0037651 & - & - & 17.24 & 16.94 & - & V22 \\
BSS 55 & 294.9576721 & -30.9620571 & - & - & 17.22 & 16.98 & - & V37 \\
BSS 56 & 295.0382690 & -30.9452572 & - & - & 17.35 & 17.00 & - & V16 \\
BSS 57 & 294.9394226 & -30.9343033 & - & - & 17.41 & 17.09 & - & V24 \\
BSS 58 & 295.0471497 & -30.9905624 & - & - & 17.49 & 17.15 & - & V17 \\
BSS 59 & 295.0498962 & -31.0348148 & - & - & 17.49 & 17.18 & - & V39 \\
BSS 60 & 295.0078125 & -30.9275074 & - & - & 17.54 & 17.23 & - & V40 \\
BSS 61 & 295.0041809 & -31.0107975 & - & - & 17.58 & 17.25 & - & V34 \\
BSS 62 & 294.9658203 & -30.9315720 & - & - & 17.65 & 17.26 & - & V23 \\
BSS 63 & 294.9459534 & -30.9596825 & - & - & 16.49 & 16.04 & - & V26 \\
BSS 64 & 295.2062378 & -30.6464367 & - & - & 17.82 & 17.41 & - & - \\
BSS 65 & 295.1806946 & -30.6018009 & - & - & 16.53 & 16.34 & - & - \\
\hline
\end{tabular}

Note. - The first 12 BSS have been identified in the WFPC2; BSS 2-26 are from the ACS observations, the first 11 being in common with the WFPC2 sample; BSS 27-65 are from the complementary WFI data set. BSS 64 and 65 lie beyond the cluster tidal radius, at $\sim 22^{\prime}$ and $24^{\prime}$ from the center, respectively, and have not been considered in the analysis of the BSS radial distribution. The last column list the corresponding SX Phe stars identified by Pych et al. (2001). 
Table 2. Number Counts of BSS, HB, and RGB Stars, and Fraction of Sampled Luminosity

\begin{tabular}{rrclcc}
\hline \hline$r_{i}{ }^{\prime \prime}$ & \multicolumn{1}{c}{$r_{e}{ }^{\prime \prime}$} & $N_{\mathrm{BSS}}$ & $N_{\mathrm{HB}}$ & $N_{\mathrm{RGB}}$ & $L^{\text {samp }} / L_{\text {tot }}^{\text {samp }}$ \\
\hline 0 & 90 & 23 & 56 & $297(1)$ & 0.23 \\
90 & 160 & 17 & 56 & $337(2)$ & 0.25 \\
160 & 250 & 12 & 56 & $325(5)$ & 0.22 \\
250 & 560 & 3 & 59 & $362(33)$ & 0.26 \\
560 & 1160 & $7(1)$ & $9(1)$ & $58(84)$ & 0.04 \\
\hline \hline
\end{tabular}

Note. - The values listed out of the parenthesis correspond to the number of stars assumed to belong to the cluster (and thus used in the analysis), while those in the parenthesis are estimated to be contaminating field stars (see Sect. 4.1). 


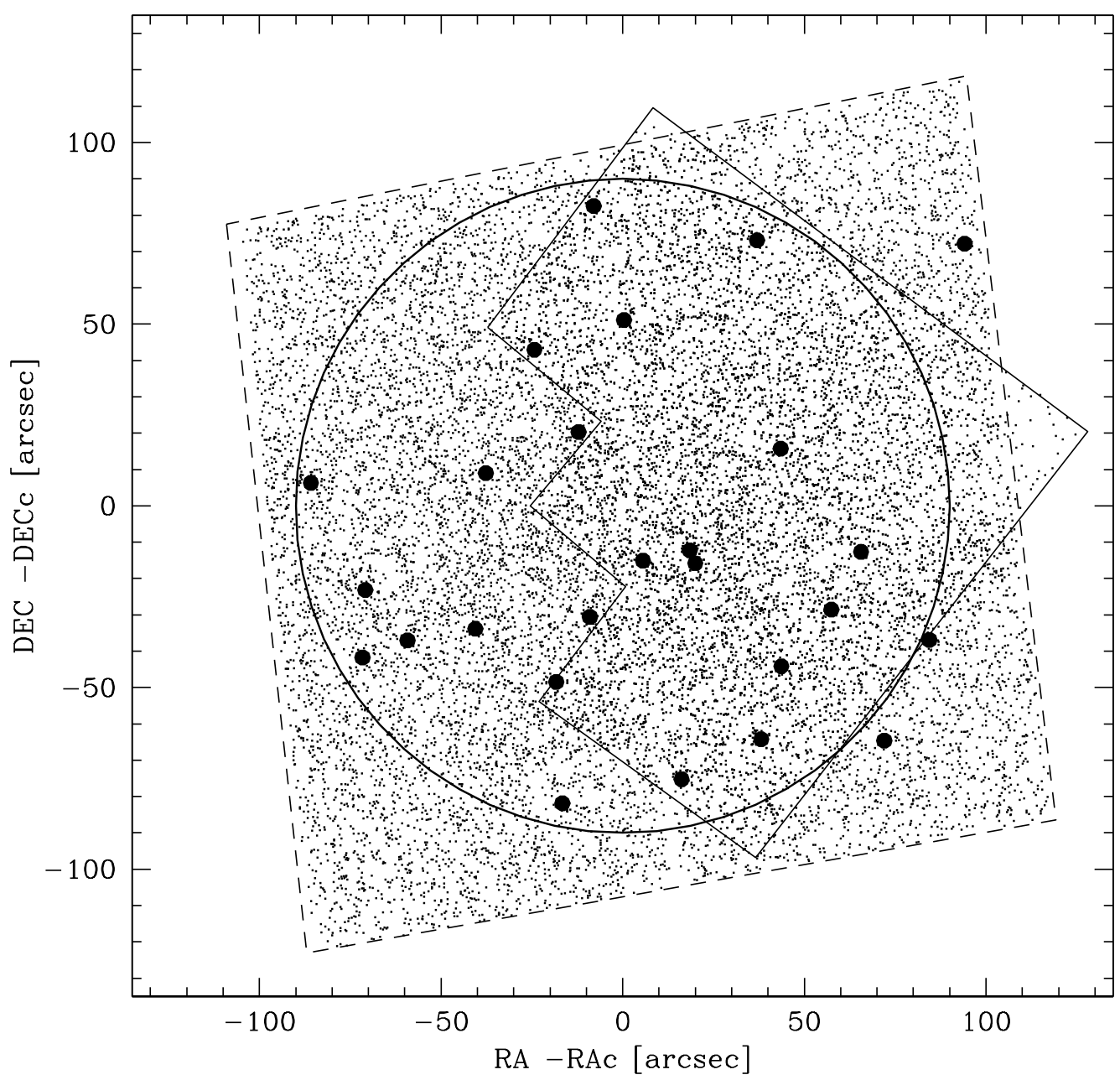

Fig. 1. - Map of the HST sample, with coordinates referred to the derived center of gravity $C_{\text {grav }}: \mathrm{RA}_{\mathrm{c}}=19^{\mathrm{h}} 39^{\mathrm{m}} 59.54, \mathrm{DEC}_{\mathrm{c}}=-30^{\mathrm{s}} 57^{\prime} 45^{\prime \prime} .14$. The solid and dashed thin lines delimit the HST-WFPC2 and HST-ACS fields of view, respectively. The selected BSS (heavy dots) and the annulus with radius $r=90^{\prime \prime}$ used to study their projected radial distribution (cfr. Table 2) are also shown. 


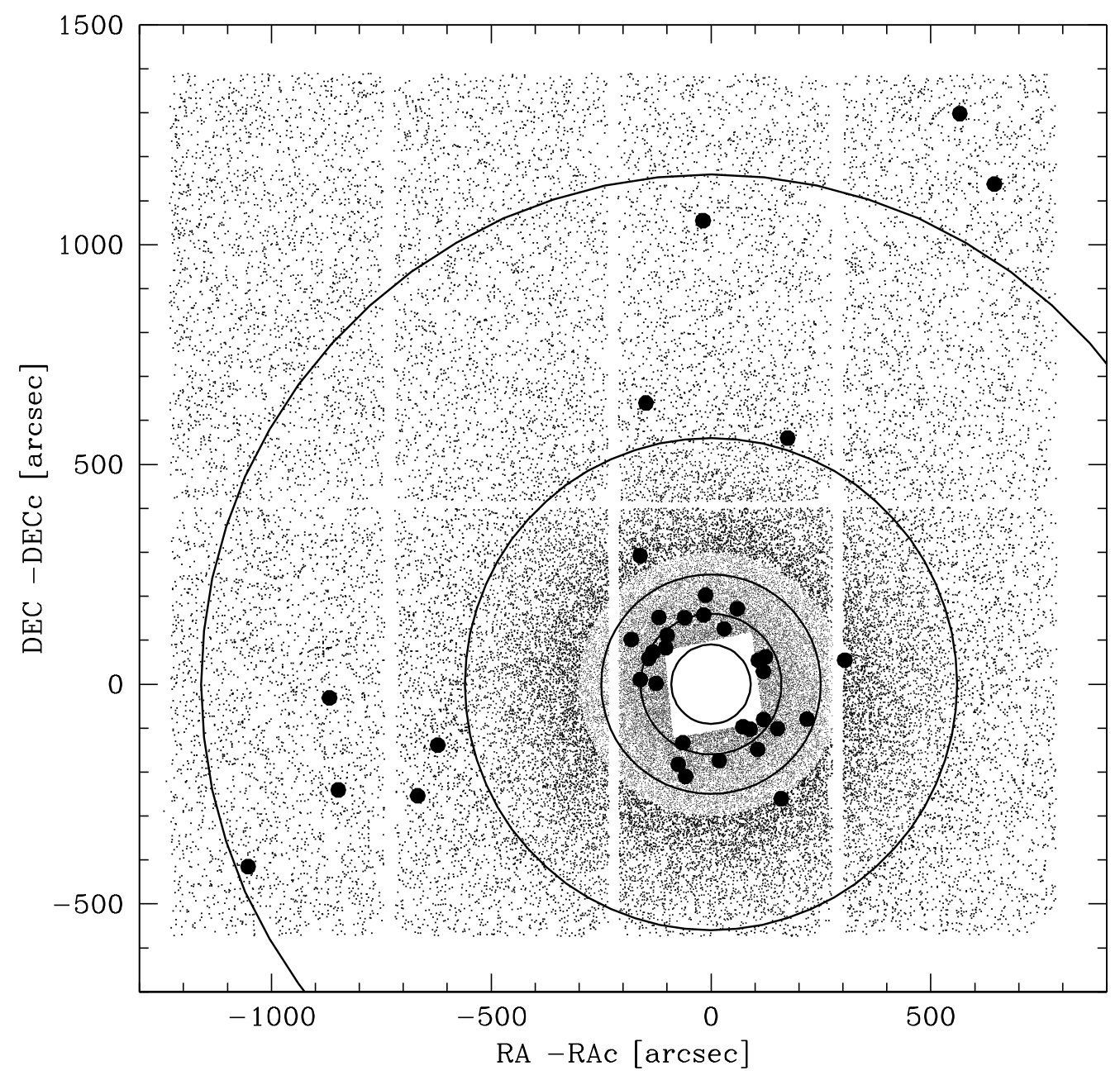

Fig. 2.- Map of the complementary WFI sample, with coordinates referred to $C_{\text {grav }}$. The empty central region corresponds to the HST-ACS FoV (dashed line in Fig. 11). All the detected BSS are marked as heavy dots, and the concentric annuli used to study their projected radial distribution (cfr. Table 2) are shown as solid circles, with the inner annulus corresponding to $r=90^{\prime \prime}$, and the outer one corresponding to the tidal radius $r_{t}=1160^{\prime \prime}$. The two candidate BSS lying beyond $r_{t}$ most probably are field stars. 


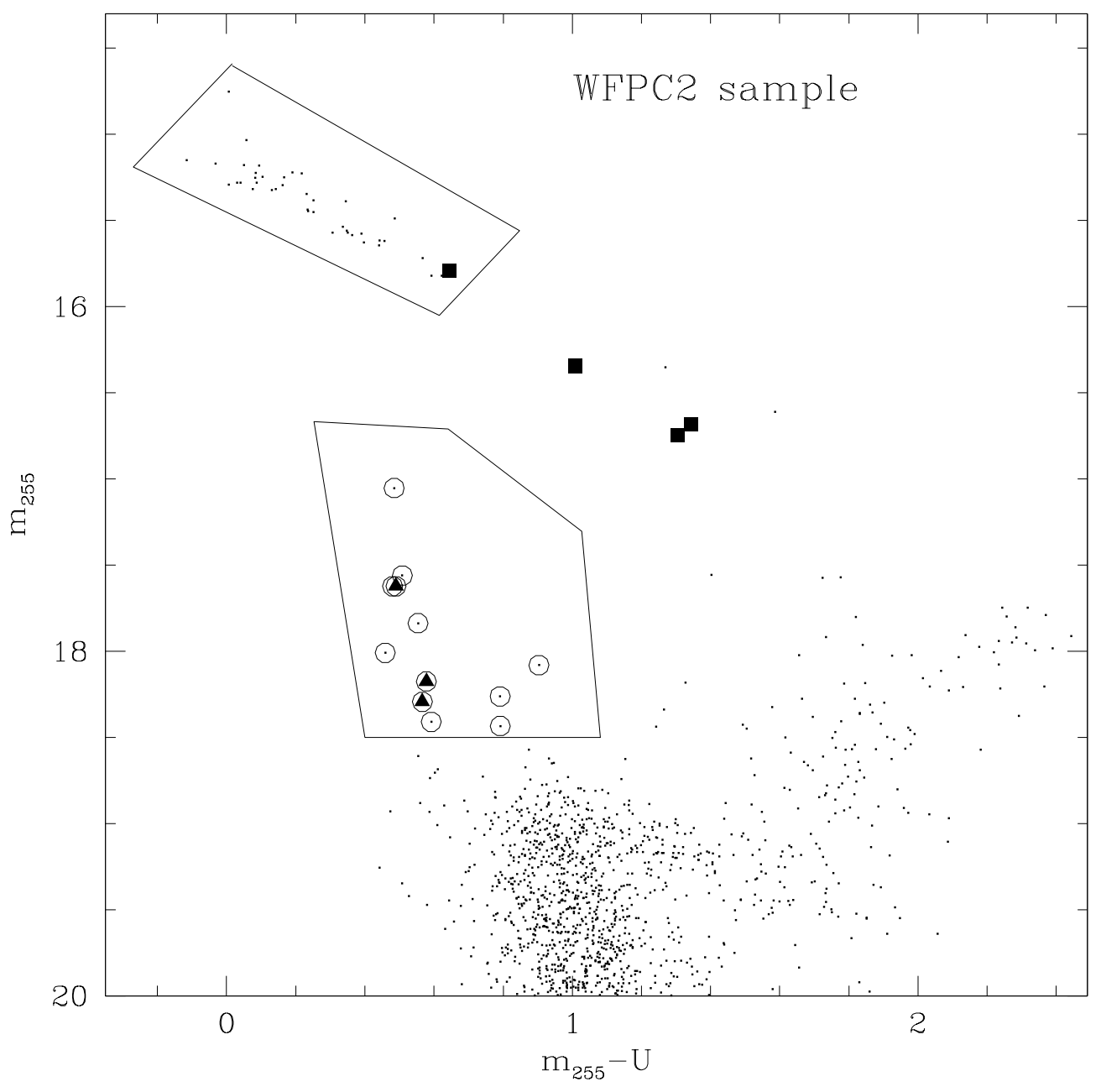

Fig. 3.- Ultraviolet CMD of the HST-WFPC2 sample. The adopted BSS and HB selection boxes are shown. The resulting fiducial BSS are marked with empty circles. Triangles correspond to the SX Phoenicis variables identified by Pych et al. (2001), while the squares mark the RRLyrae identified by Olech et al. (1999) and included in our HB sample. 


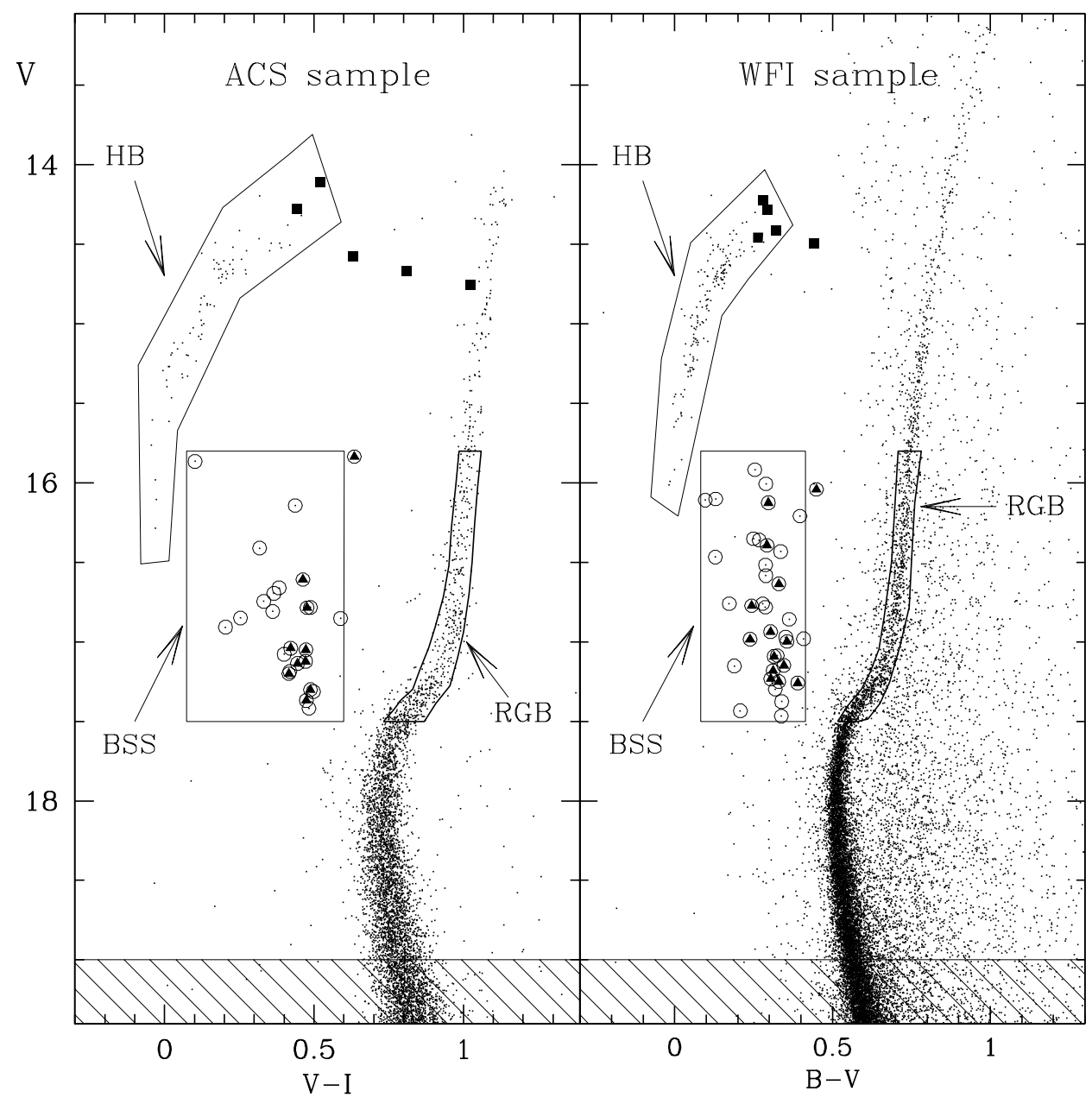

Fig. 4.- Optical CMDs of the HST-ACS and of the complementary WFI samples. The adopted BSS, HB, and RGB selection boxes are shown. Symbols are as in Figure 3. The hatched regions indicate the magnitude limit $(V \leq 19)$ adopted for the computation of the cluster surface density profile. 


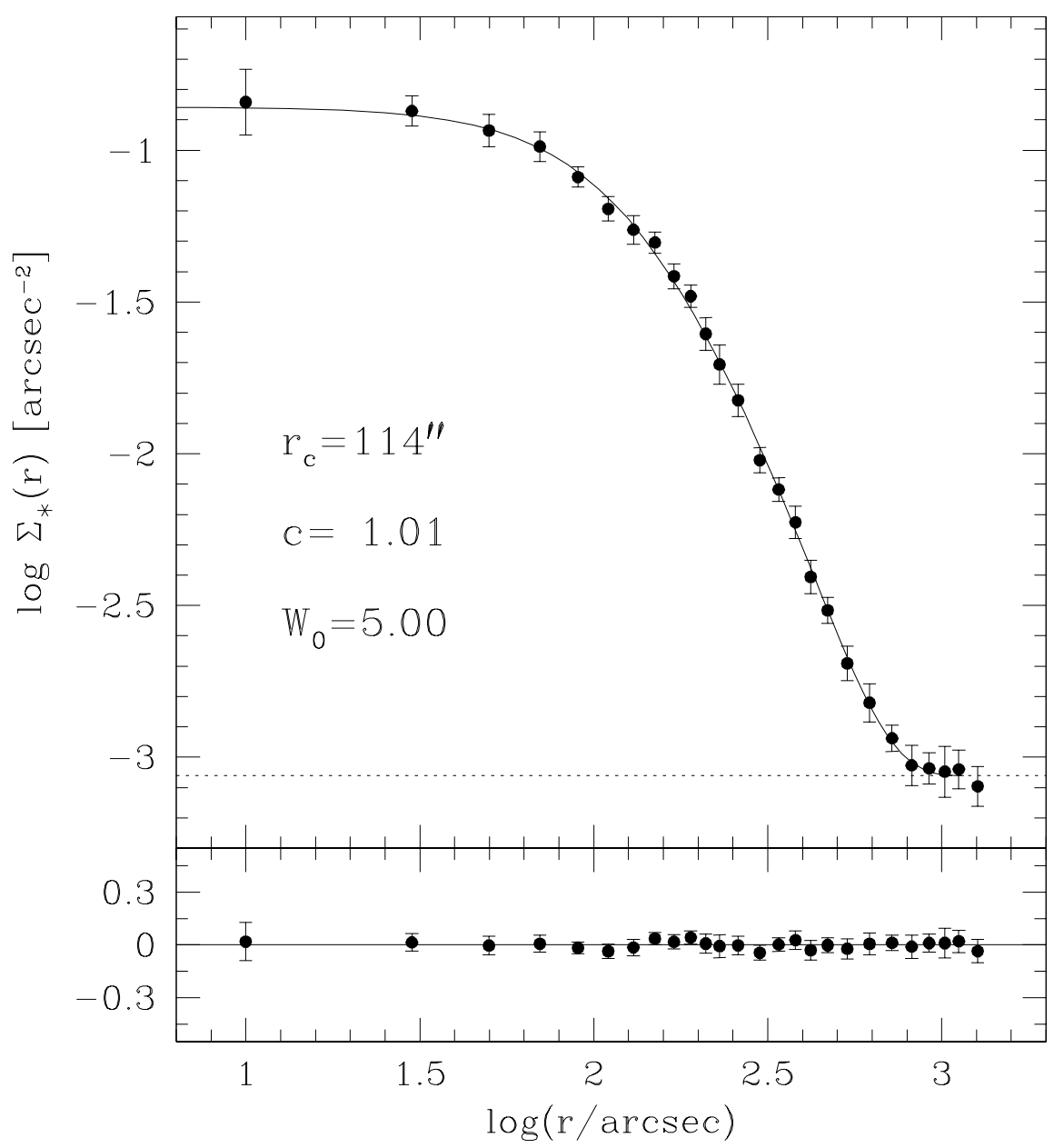

Fig. 5.- Observed surface density profile (dots and error bars) and best-fit King model (solid line). The radial profile is in units of number of stars per square arcseconds. The dotted line indicates the adopted level of the background, and the model characteristic parameters (core radius $r_{c}$, concentration $c$, dimensionless central potential $W_{0}$ ) are marked in the figure. The lower panel shows the residuals between the observations and the fitted profile at each radial coordinate. 


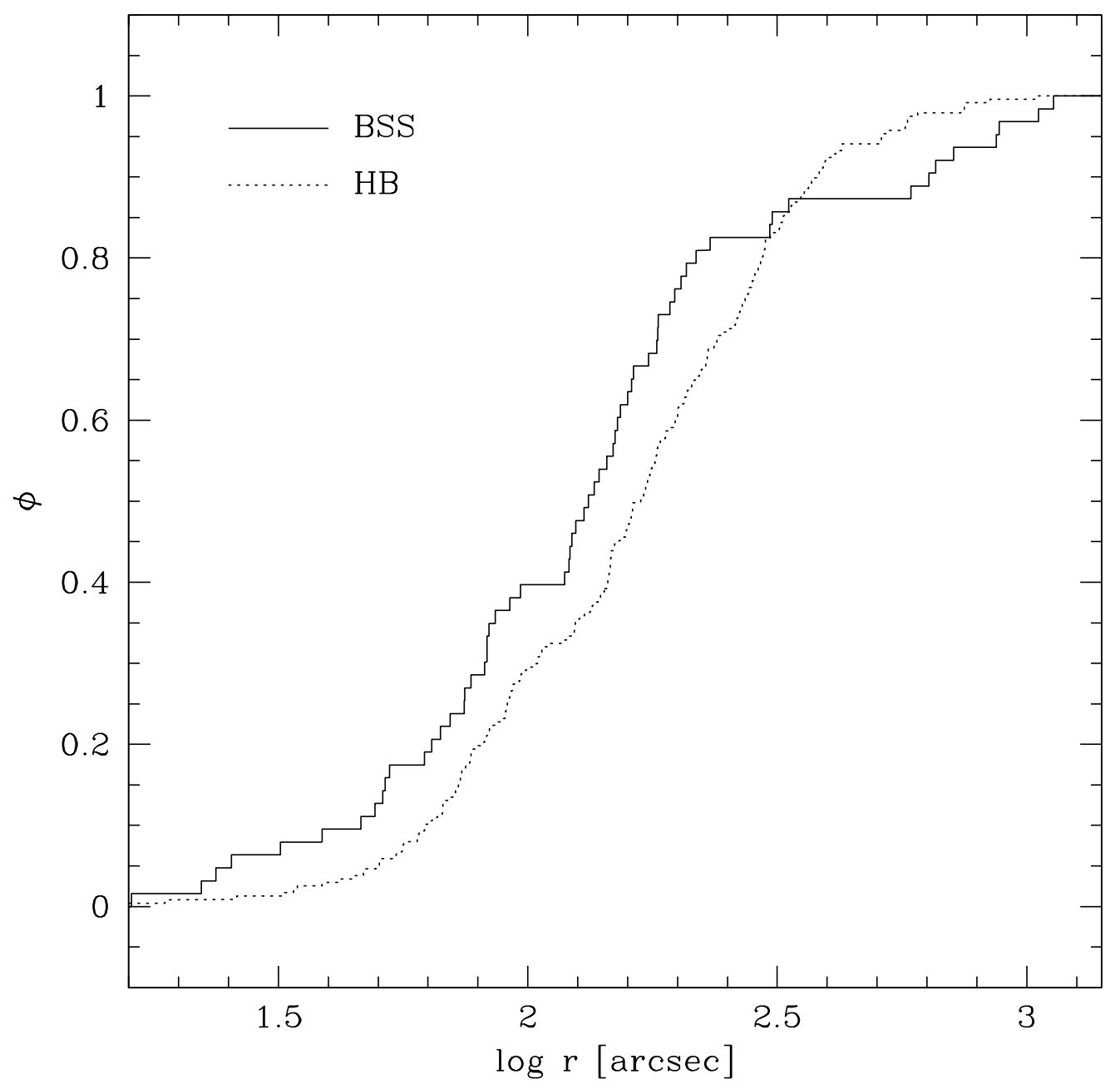

Fig. 6.- Cumulative projected radial distribution of BSS (solid line) and HB stars (dotted line). 


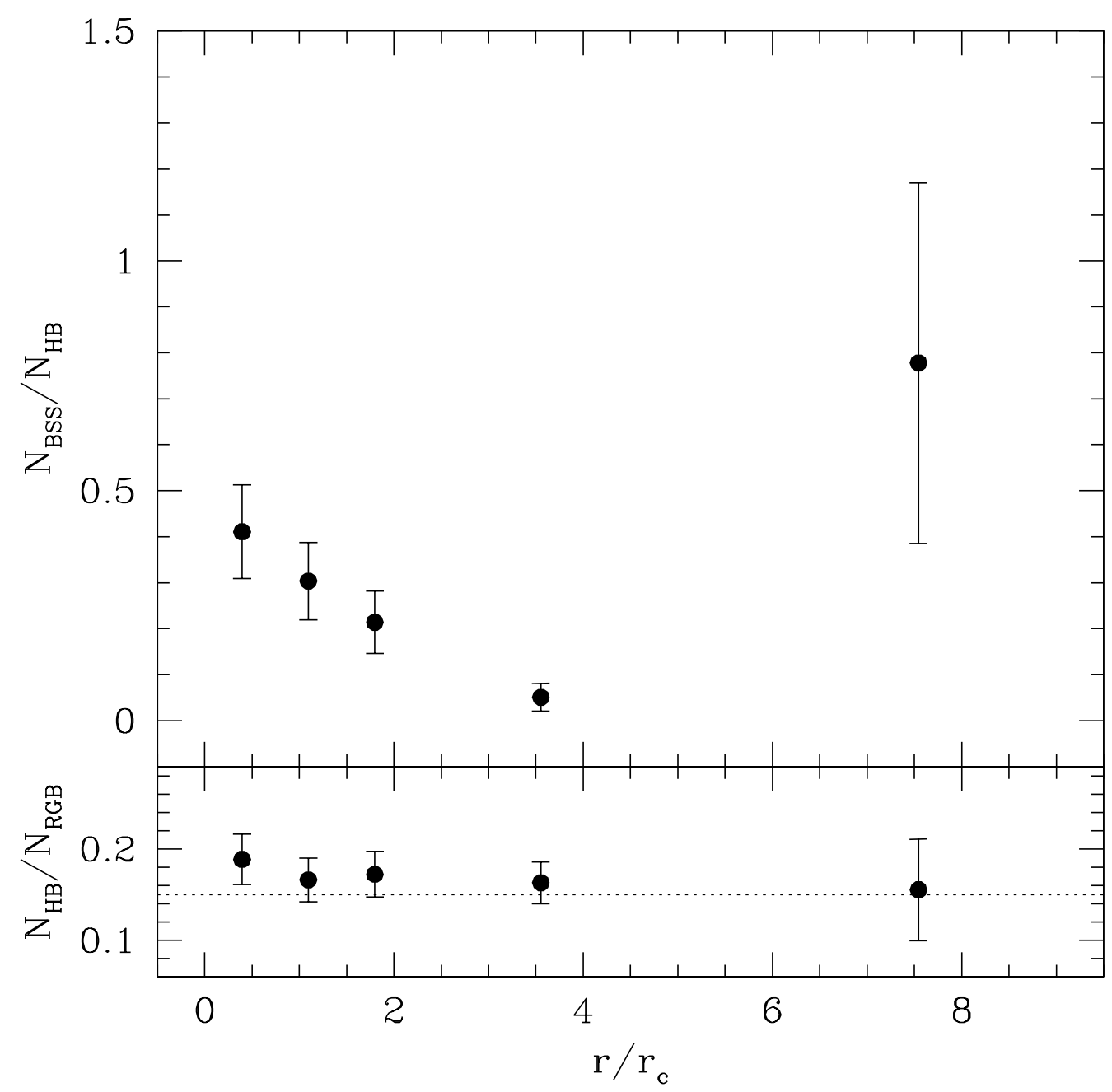

Fig. 7.- Upper panel: Projected radial distribution of the specific frequency $N_{\mathrm{BSS}} / N_{\mathrm{HB}}$, as a function the radial distance from the cluster center, expressed in units of the core radius. Lower panel: The same as above, for the specific frequency $N_{\mathrm{HB}} / N_{\mathrm{RGB}}$. The dotted line corresponds to the value $(\sim 0.15)$ predicted by the population synthesis models of Pietrinferni et al. (2006) for the ratio between the evolutionary time-scales of the HB and RGB (in the range $15.8 \leq V \leq 17.5$ ) phases. 


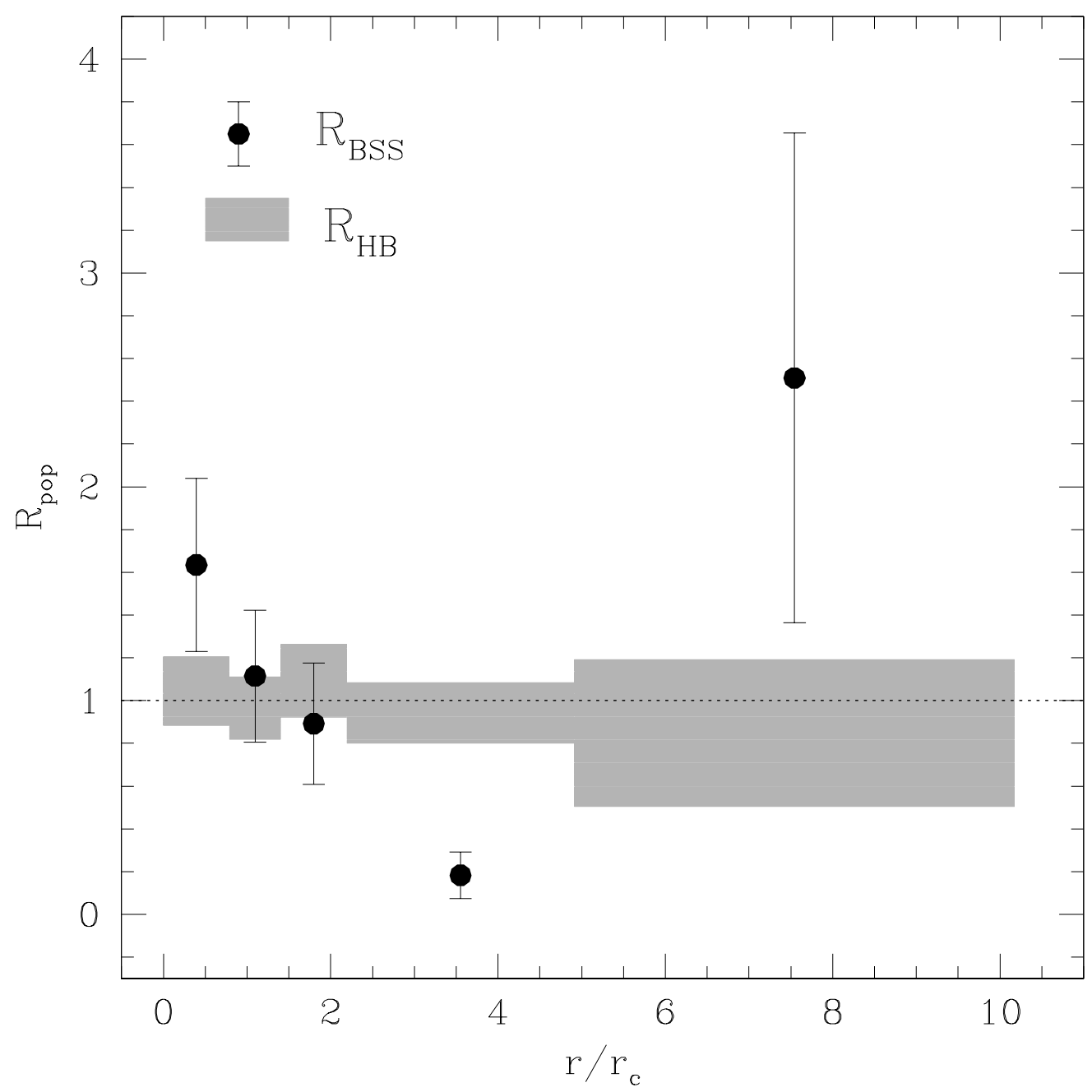

Fig. 8.- Projected radial distribution of the double normalized ratios of BSS (dots) and HB stars (gray rectangles), as defined in equation (1). The error bars (represented by the vertical sizes of the rectangles in the case of $R_{\mathrm{HB}}$ ) are computed as described in Sabbi et al. (2004). The dotted line corresponds to the value $\left(R_{\mathrm{pop}}=1\right)$ expected for any normal post-MS population in the cluster (see Sect.51). 